Cahiers $d u$ MONDE RUSSE

\section{Cahiers du monde russe}

Russie - Empire russe - Union soviétique et États indépendants

$53 / 4 \mid 2012$

Varia

\title{
Klaus-Michael Mallmann, Andrej Angrick, Jürgen Matthäus, Martin Cüppers, éds., Die « Ereignismeldungen UdSSR » 1941
}

\section{Nathalie Moine}

\section{(2) OpenEdition}

12 Journals

Édition électronique

URL : http://journals.openedition.org/monderusse/7723

DOI : 10.4000/monderusse. 7723

ISSN : $1777-5388$

Éditeur

Éditions de l'EHESS

Édition imprimée

Date de publication : 15 décembre 2012

ISSN : 1252-6576

Référence électronique

Nathalie Moine, « Klaus-Michael Mallmann, Andrej Angrick, Jürgen Matthäus, Martin Cüppers, éds., Die «Ereignismeldungen UdSSR » 1941 », Cahiers du monde russe [En ligne], 53/4 | 2012, mis en ligne le 02 décembre 2013, Consulté le 25 septembre 2020. URL : http://journals.openedition.org/monderusse/ 7723 ; DOI : https://doi.org/10.4000/monderusse. 7723

Ce document a été généré automatiquement le 25 septembre 2020.

(c) École des hautes études en sciences sociales 


\title{
Klaus-Michael Mallmann, Andrej Angrick, Jürgen Matthäus, Martin Cüppers, éds., Die « Ereignismeldungen UdSSR » 1941
}

\author{
Nathalie Moine
}

\section{RÉFÉRENCE}

Klaus-Michael MALLMANN, Andrej ANGRICK, Jürgen MATTHÄUS, Martin CÜPPERS, éds., Die «Ereignismeldungen UdSSR » 1941. Dokumente der Einsatzgruppen in der Sowjetunion. Darmstadt : Wissenschaftliche Buchgesellschaft, 2011, 925 p.

1 Le présent recueil de documents, regroupant l'ensemble des rapports d'activités des Einsatzgruppen en URSS (Ereignismeldungen UdSSR) pour l'année 1941, constitue un événement puisqu'ils sont publiés pour la première fois dans leur intégralité. En effet, alors même qu'ils furent utilisés dès l'immédiat après-guerre à des fins judiciaires, notamment lors du procès des Einsatzgruppen qui se tint à Nuremberg à la fin des années 1940, et qu'ils étaient consultables par les spécialistes du nazisme en divers lieux, seules des bribes en avaient été traduites et livrées au public, dans des éditions parfois peu scrupuleuses. Projeté dès les années 1950, cet ambitieux projet n'a donc vu le jour que très récemment, le présent volume devant être suivi de trois autres publications, afin de couvrir l'ensemble de la documentation en provenance des Einsatzgruppen en Union soviétique.

2 Les Ereignismeldungen sont d'abord rédigés quasi quotidiennement, le premier rapport étant daté du 23 juin 1941, le dernier, numéroté 195, datant du 24 avril 1942 (le présent volume comprend les rapports 1 à 149, ce dernier étant daté du 22 décembre 1941). Leur succèdent 55 rapports hebdomadaires, (Meldungen aus den besetzten Ostgebieten), qui s'interrompent après le n 55, daté du 21 mai 1943. 
Une introduction serrée offre une critique de source particulièrement salutaire pour les non spécialistes. Les auteurs rappellent ainsi que l'écriture de ces rapports, à Berlin, dans les bureaux du RSHA (Reichssicherheitshauptamt, la centrale en charge de l'ensemble des organes responsables de la sécurité du Reich), répondaient à la fois, de la part de leurs commanditaires, à un besoin d'informations sur le déroulement quotidien des activités de ces petits groupes lâchés à l'arrière de la Wehrmacht pour "nettoyer " le pays conquis, mais aussi à une concurrence aigüe entre administrations nazies, sans compter l'effet de cohésion interne nécessaire à une institution récente composée de corps aux pratiques et traditions différentes. Ces enjeux de pouvoir ne pouvaient donc qu'influer sur leur rédaction.

Les responsables de cette édition se livrent également à une étude passionnante sur le processus concret de rédaction de ces rapports, des informations envoyées par les commandos aux nouvelles reçues par Berlin, en passant par les quartiers généraux des Einsatzgruppen situés en territoire soviétique occupé. Ils montrent ainsi que beaucoup de questions subsistent sur ce travail de sélection et de réécriture des informations primaires, à la fois du fait de la destruction massive des archives et du caractère douteux des dépositions d'après-guerre.

5 Pour cet ensemble de raisons, les faits présents dans les rapports finaux ne sont donc pas à prendre forcément au pied de la lettre, y compris les chiffres des victimes. L'impressionnant appareil critique qui accompagne chaque rapport permet alors à ces excellents spécialistes de préciser, dans la mesure du possible, ce que d'autres sources allemandes peuvent nous dire, notamment sur d'autres massacres non signalés dans les rapports. L'essentiel du personnel dirigeant de ces groupes fait également l'objet de notes qui déroulent leur biographie, de leurs années de formation à leur destin d'aprèsguerre, accompagnées des références d'archives. Le fait que les rapports soient publiés intégralement permet d'élargir considérablement notre compréhension de cette séquence fondamentale dans la destruction des juifs d'Europe, et c'est bien l'objectif clairement affiché par les responsables de ce projet éditorial qui souhaitent ainsi favoriser l'insertion de l'étude de la Shoah à l'Est dans un contexte de violences plus large, mais aussi revenir sur l'univers mental de ses auteurs, dans lequel la haine du « judéobolchevisme » était à la fois centrale et articulée à toutes sortes de thématiques, qu'on pourrait résumer dans l'obsession de mettre en place une Volkstumpolitik, c'est-àdire une politique d'aménagement $d u$ territoire soviétique fondée sur des préoccupations raciales, attentives aux particularités des différentes populations rencontrées sur le sol soviétique ${ }^{1}$. À bon droit, les auteurs rappellent donc que la lecture de ces rapports peut tout aussi bien concerner des spécialistes d'autres questions que le génocide, tant les observations (ou élucubrations) fourmillent et contrastent avec le caractère lapidaire des informations concernant les massacres proprement dits, de façon encore plus saisissante que n'avaient pu le faire des éditions précédentes qui sélectionnaient uniquement les passages directement relatifs à la Shoah. Car c'est bien une des pages essentielles de la Shoah à l'Est qui est ici documentée, même si le terme même de Shoah, amplement justifié a posteriori, masque la nature des actions menées à l'Est par les Einsatzgruppen. Les auteurs rappellent ainsi que les tueries ne répondirent pas un ordre qui aurait été donné d'en haut à la veille de l'invasion, mais que les rapports témoignent d'un processus de radicalisation tout en y participant, du fait notamment qu'ils font circuler des chiffres de victimes de plus en plus inouïs, mettant en concurrence les différentes unités nazies présentes en URSS. La 
centralité de la Shoah se traduit dans l'appareil de notes, on l'a dit particulièrement soigné, même si on pourrait regretter l'absence de dialogue avec l'historiographie fondée sur des sources soviétiques (à ne pas confondre avec les archives en provenance de fonds soviétiques mais produites par les administrations nazies, dont les auteurs sont d'excellents connaisseurs). L'introduction mentionne furtivement le travail d'enquête des autorités est-européennes, notamment soviétiques, mais la confrontation est visiblement repoussée à des phases ultérieures de l'occupation. Pourtant, cette indifférence peut amener à des conclusions hâtives, en particulier l'affirmation selon laquelle ces rapports sont les uniques preuves documentaires existantes pour des " petits massacres ». L'exemple donné étant les tueries perpétrées par le SK 4a, relevant de l'Einsatzgruppe C, dans les localités ukrainiennes de Gornostaipol' et Dymer ${ }^{2}$, une simple consultation de l'encyclopédie dirigée par Il'ja Al'tman, qui recense précisément l'ensemble des lieux de massacres connus en Union soviétique sur la base des enquêtes menées par les Soviétiques, dément cette affirmation ${ }^{3}$. S'il est évident que les données des Soviétiques appellent à une manipulation extrêmement prudente, leur croisement avec les données allemandes paraît donc incontournable, et on pourrait se demander pourquoi la méfiance des auteurs du projet face aux informations en provenance des Soviétiques tombe, inexplicablement, lorsqu'il s'agit du Livre Noir dirigée par Il'ja Erenburg et Vasilij Grossman, dont la version parue dans les années 1990 et traduite dans plusieurs langues, est citée à plusieurs reprises sans aucun caveat. L'absence d'index géographique est d'ailleurs révélatrice, puisqu'elle rend très difficile ce croisement, et dessert par ailleurs l'énorme travail d'informations effectué pour plusieurs localités, qui apparaît sous forme de note de bas de page à la première occurrence, pour cette même raison difficile à repérer, du lieu. En revanche, les nombreuses cartes sont à la hauteur d'un projet éditorial exigeant qui facilitera grandement, malgré tout, la coopération possible entre spécialistes et satisfera la curiosité du plus grand nombre pour ce qui reste un des plus étonnants " autotémoignages ${ }^{4}$ des crimes nazis.

\section{NOTES}

1. Sur tout ceci, cf. Christian Ingrao, Croire et détruire: Les intellectuels dans la machine de guerre SS, P. : Fayard, 2010.

2. Klaus-Michael Mallmann et alii, éds., Die « Ereignismeldungen UdSSR »1941..., p. 8.

3. Il'ja Al'tman, Holokost na territorii SSSR: Enciklopedija, M. : Rossspen, 2009, notice « Gornostaipol '", p. 228-229 et "Dymer", p. 287-288.

4. Mallmann et alii, éds., Die « Ereignismeldungen UdSSR »1941..., p. 7. 